\title{
Lenalidomide for the treatment of relapsed and refractory multiple myeloma
}

This article was published in the following Dove Press journal:

Cancer Management and Research

13 August 2012

Number of times this article has been viewed

\author{
Niels WCJ van de Donk ${ }^{1,2}$ \\ Güllü Görgün' \\ Richard WJ Groen ${ }^{1,3}$ \\ Jana Jakubikova' \\ Constantine S Mitsiades' \\ Teru Hideshima' \\ Jacob Laubach' \\ Inger S Nijhof ${ }^{2}$ \\ Reinier A Raymakers ${ }^{2}$ \\ Henk M Lokhorst ${ }^{2}$ \\ Paul G Richardson' \\ Kenneth C Anderson' \\ 'Department of Medical Oncology, \\ Dana-Farber Cancer Institute, \\ Harvard Medical School, Boston, MA, \\ USA; '2Department of Hematology, \\ ${ }^{3}$ Department of Cell Biology, \\ University Medical Center Utrecht, \\ Utrecht, The Netherlands
}

Correspondence: Güllü Görgün

Dana-Farber Cancer Institute, 450

Brookline Ave M557, Boston, MA,

02215, USA

Tel + I 6176326553

Fax +l 61 76322140

Email gullu_gorgun@dfci.harvard.edu

Niels WCJ van de Donk

Department of Hematology

University Medical Center Utrecht,

Heidelberglaan 100, 3584CX, Utrecht,

The Netherlands

$\mathrm{Tel}+3 \mathrm{I} 887555555$

Fax +3 I 30252374 I

Email n.w.c.j.vandedonk@umcutrecht.nl

\begin{abstract}
Lenalidomide is an amino-substituted derivative of thalidomide with direct antiproliferative and cytotoxic effects on the myeloma tumor cell, as well as antiangiogenic activity and immunomodulatory effects. Together with the introduction of bortezomib and thalidomide, lenalidomide has significantly improved the survival of patients with relapsed and refractory myeloma. The most common adverse events associated with lenalidomide include fatigue, skin rash, thrombocytopenia, and neutropenia. In addition, when lenalidomide is combined with dexamethasone or other conventional cytotoxic agents, there is an increase in the incidence of venous thromboembolic events. There is now evidence that continued treatment with lenalidomide has a significant impact on survival by improving the depth and duration of response. This highlights the value of adverse event management and appropriate dose adjustments to prevent toxicity, and of allowing continued treatment until disease progression. In this review, we will discuss the different lenalidomide-based treatment regimens for patients with relapsed/ refractory myeloma. This is accompanied by recommendations of how to manage and prevent adverse events associated with lenalidomide-based therapy.
\end{abstract}

Keywords: lenalidomide, multiple myeloma, immunomodulatory drugs, relapse treatment, refractory disease

\section{Introduction}

Lenalidomide and pomalidomide, as more potent derivatives of thalidomide, have been found to be less toxic and more active than their parent drug in the treatment of multiple myeloma (MM). ${ }^{1-4}$ Because of the structural similarities between thalidomide and its derivative immunomodulatory drugs (IMiDs), these agents have similar modes of action with both direct antitumor activity and indirect immunomodulatory and antiangiogenic effects (Figure 1). IMiDs directly kill MM cells by the induction of cell cycle arrest and caspase-dependent apoptosis. ${ }^{5-7}$ In addition, we have shown that IMiDs target a subpopulation of MM cells with stem cell-like features (ie, sidepopulation cells). ${ }^{8}$ Recently, it has been demonstrated that cereblon, the primary target for thalidomide teratogenicity, ${ }^{9}$ is required for the cytotoxic effects of thalidomide, lenalidomide, and pomalidomide. ${ }^{10}$

In addition, lenalidomide also impairs MM cell survival and proliferation through interference with the protective properties of bone marrow stromal cells, including the down-regulation of adhesion molecules such as VCAM-1 and ICAM-1, ${ }^{11,12}$ and inhibition of the production of cytokines like IL-6 and TNF- $\alpha .{ }^{13}$ Furthermore, IMiDs inhibit angiogenesis by downregulation of vascular endothelial growth factor and $\beta$-fibroblast growth factor ${ }^{14,15}$ and impair osteoclastogenesis by reducing RANKL levels. ${ }^{16}$ IMiDs also 


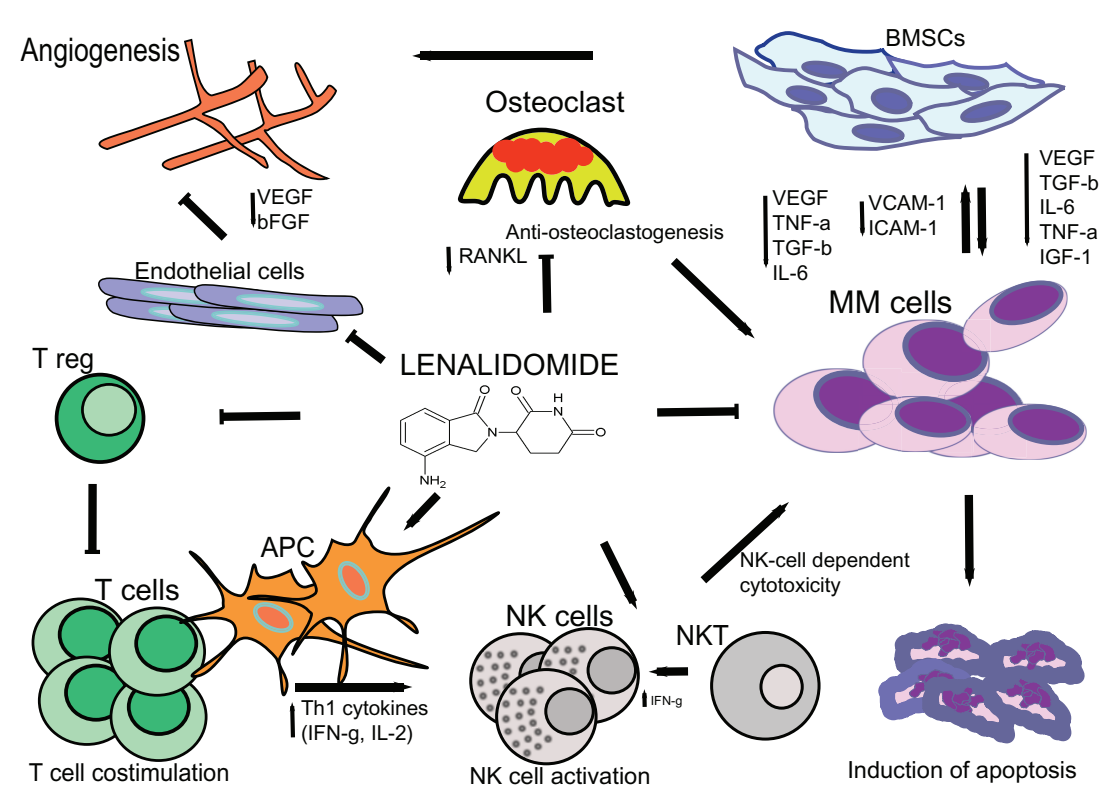

Figure I The proposed mechanisms of action of lenalidomide in multiple myeloma include immune modulation (A); interference with tumor microenvironment interactions (B); and direct antitumor effects (C). (A) Immunomodulation by lenalidomide includes T cell co-stimulation, suppression of Tregs, increased production of ThI cytokines, and activation of NK and NKT cells. (B) Lenalidomide mediates disruption of myeloma cell-microenvironment interactions via several mechanisms including antiangiogenesis, anti-inflammatory effects, antiosteoclastogenic properties, modulation of cytokine production, and downregulation of adhesion molecules. (C) IMiDs also exert direct effects on myeloma cells via cell cycle arrest and induction of apoptosis.

Abbreviations: T reg, regulatory T cell; BMSCs, bone marrow stromal cells; APC, antigen-presenting cell; NK cells, natural killer cells; NKT cells, natural killer T cells; ICAM-I, intercellular adhesion molecule I;VCAM-I, vascular cell adhesion molecule I;VEGF, vascular endothelial growth factor; RANKL, receptor activator of nuclear factor kappa-B ligand; bFGF, basic fibroblast growth factor;TGF- $\beta$, transforming growth factor- $\beta$; IGF-I, insulin-like growth factor I; TNF- $\alpha$, tumor necrosis factor $\alpha$; IL-6, interleukin-6.

have immunomodulatory effects including stimulation of T cell proliferation, increased production of IL-2 and IFN $\gamma,{ }^{13}$ and enhancement of cytotoxic T lymphocyte, natural killer T, and natural killer effector cell activity against MM cells..$^{17,18}$ Lenalidomide is more potent than thalidomide in both stimulating $\mathrm{T}$ cell proliferation via the $\mathrm{T}$ cell receptor and in enhancing IL-2 and IFN $\gamma$ production. IMiDs also decrease the development of regulatory T cells in MM. ${ }^{19}$ In addition, lenalidomide inhibits myeloid cell-mediated inflammatory function by decreasing the secretion of IL-6, TNF $\alpha$, and IL-10. ${ }^{20}$ We have demonstrated that IMiDs induce immune effector cell activation by triggering positive costimulatory molecule CD28 signaling in T cells, ${ }^{17,21}$ as well as regulate cytokine signaling by downregulating the suppressor of cytokine signaling (SOCS)1 in immune effector cells in MM, thereby inducing IL-2 and IFN $\gamma$ production. ${ }^{22}$

In this review, we will discuss the clinical activity and optimal use of lenalidomide and lenalidomide-based combinations in the management of relapsed and refractory MM.

\section{Single-agent lenalidomide in relapsed/refractory $M M$}

Single-agent lenalidomide was shown to be effective and well tolerated in relapsed/refractory MM patients who had received a median of three prior regimens as part of a Phase I trial in which the maximum tolerated dose (MTD) was found to be $25 \mathrm{mg}$ daily, and $29 \%$ of the patients obtained at least a partial response (PR). ${ }^{4}$ Importantly, no significant somnolence, constipation, or neuropathy was observed. The most common adverse events included fatigue, skin rash, thrombocytopenia, and neutropenia, which proved manageable with dose-reduction and granulocyte colony stimulating factor (G-CSF) support. ${ }^{4}$ Single-agent lenalidomide did not significantly increase the risk of venous thromboembolism (VTE). ${ }^{4}$ Other studies have since confirmed that single-agent lenalidomide has a favorable tolerability and promising efficacy, even after prior treatment with thalidomide, bortezomib, and/or high-dose melphalan with autologous stem cell rescue. ${ }^{23,24}$

\section{Lenalidomide plus dexamethasone in relapsed/refractory $M M$}

In vitro studies demonstrated that dexamethasone enhances the anti-MM effects of lenalidomide. ${ }^{5,6}$ Based on these preclinical and early phase clinical trial data suggesting that response rates can be markedly enhanced by the addition of dexamethasone, two randomized Phase III trials (MM-009 and MM-010) compared lenalidomide (25 mg on days 1-21 
of a 28-day cycle) plus dexamethasone (40 mg on days $1-4$, 9-12, and 17-20 for the first four cycles, and $40 \mathrm{mg}$ on days 1-4 thereafter) with placebo plus dexamethasone in relapsed/ refractory MM patients who had received a median of two previous therapies (Table 1). Dimopoloulos et $\mathrm{a}^{25}$ demonstrated the superior efficacy of lenalidomide-dexamethasone compared with placebo plus dexamethasone in terms of higher overall response rate (complete response (CR) + PR; 60.2\% vs $24.0 \% ; P<0.001)$ and $\mathrm{CR}$ rate $(15.9 \%$ vs $3.4 \% ; P<0.001)$. The authors also noted that lenalidomide-dexamethasone exhibited a longer median time to progression (TTP) (11.3 vs 4.7 months; $P<0.001$ ) and median overall survival (OS) (not reached and 20.6 months; $P=0.03$ ) when compared with placebo plus dexamethasone. In the study of Weber et $a{ }^{26}$ comparable results were reported with a superior response rate ( $\geq$ PR: $61.0 \%$ vs $19.9 \% ; P<0.001$ ), CR rate $(14.1 \%$ vs $0.6 \%$; $P<0.001$ ), median TTP (11.1 vs 4.7 months; $P<0.001$ ), and median OS (29.6 vs 20.2 months; $P<0.001)$ in the lenalidomide-dexamethasone group when compared to the placebo-dexamethasone group. Adverse events associated with lenalidomide therapy were neutropenia, thrombocytopenia, and thromboembolic complications in both studies. ${ }^{25,26}$ There was a non-significant trend toward increased grade 3 or 4 infections in lenalidomide recipients. ${ }^{25,26}$

Pooled analysis of both randomized Phase III trials with an extended median follow-up of 48 months demonstrated a continued prolongation of overall survival for the lenalidomide-dexamethasone group (38.0 months) versus the dexamethasone single-agent group (31.6 months), despite a crossover of $48 \%$ of the patients to either lenalidomide or lenalidomide plus dexamethasone as subsequent salvage therapies. ${ }^{27}$

Response to lenalidomide plus dexamethasone improved over time, with better quality of response associated with improved clinical outcomes. Median TTP and OS were longer in patients who achieved $\mathrm{CR} /$ very good partial response (VGPR) compared to patients who obtained a PR (TTP: 27.7 vs 12.0 months; OS: not yet reached vs 44.2 months). ${ }^{28}$ San Miguel et al showed that patients who participated in MM-009 or MM-010 who achieved PR or better and continued therapy had an overall survival of 50.9 months, compared to 35.0 months in patients who discontinued lenalidomidedexamethasone due to adverse events, withdrawal of consent, or other reasons. ${ }^{29,30}$ This suggests that continued treatment has a significant impact on survival, possibly by improving the depth of response.

Preliminary results from another pooled analysis of both Phase III studies showed that patients whose dexamethasone

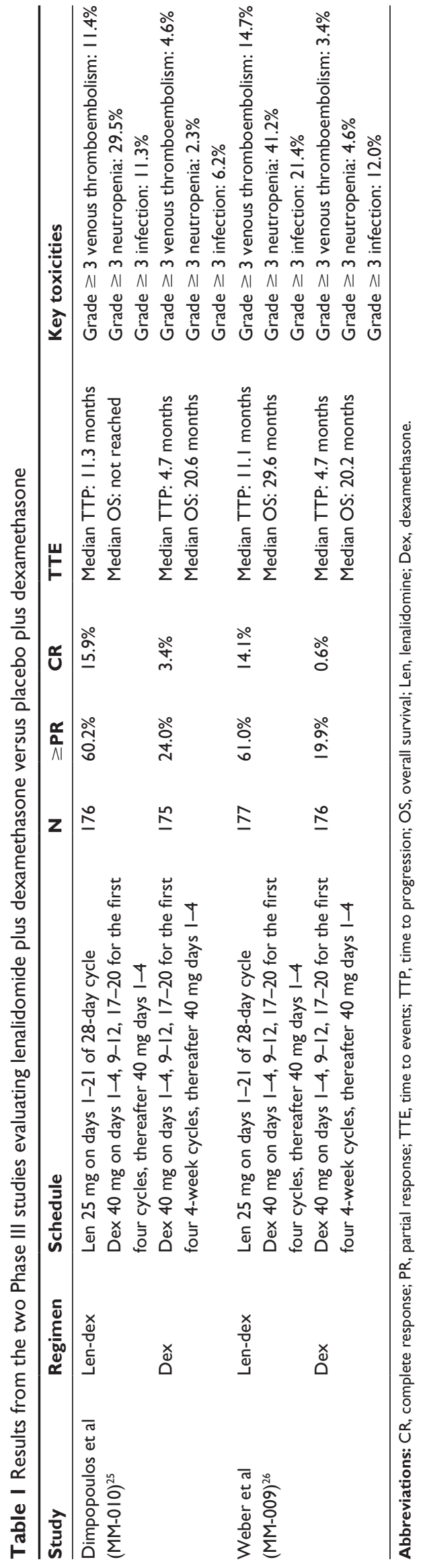


dose was reduced because of toxicity had a better outcome compared with patients who continued on high-dose dexamethasone. ${ }^{31}$ In newly diagnosed MM, lenalidomide combined with low-dose dexamethasone was also associated with lower toxicity and better overall survival than lenalidomide with high-dose dexamethasone, ${ }^{32}$ this has been widely adopted, even in the relapsed/refractory MM setting. An appropriate approach to minimize treatment-related toxicity in case of an aggressive relapse (eg, with hypercalcemia or renal failure) is to start with high-dose dexamethasone for rapid disease control, which can be followed by low-dose dexamethasone in case of response. Another subanalysis showed that patients with progression-free survival (PFS) $\geq 12$ months who had dose reductions of lenalidomide after $\geq 12$ months had better PFS than those who had earlier dose reductions or no dose reductions. ${ }^{33}$ This suggests that it is important to continue fulldose lenalidomide therapy for at least 12 months in patients who tolerate the treatment, and after this time, the dose can be reduced without compromising treatment efficacy. Overall, these studies highlight the value of adverse event management and appropriate dose adjustments to prevent toxicity, thereby allowing continued treatment until disease progression.

\section{Efficacy of lenalidomide plus dexamethasone and previous treatment}

Stadtmauer et $\mathrm{al}^{34}$ showed in a subset analysis of both Phase III trials that lenalidomide-dexamethasone was more effective in terms of response, TTP, PFS, and OS in patients who had one prior therapy when compared to those who had two or more earlier therapies, indicating that the greatest benefit occurs with early use of lenalidomide-dexamethasone in relapsed/refractory MM. Another pooled analysis of all patients that participated in these randomized trials showed that lenalidomide-dexamethasone was more effective than dexamethasone alone in both thalidomide-exposed and naïve patients. ${ }^{35}$ However, higher efficacy in terms of overall response rate, TTP, and PFS of the combination of lenalidomide-dexamethasone or dexamethasone alone was observed in thalidomide-naïve patients compared to thalidomide-exposed patients, suggesting some degree of cross-resistance between thalidomide and lenalidomide. However, patients previously treated with thalidomide had significantly more prior lines of therapy compared with patients who were thalidomide naïve. ${ }^{35}$ A French study showed similar results with inferior TTP and OS for patients that previously progressed on thalidomide. ${ }^{36}$ In contrast, an Italian study showed that response, PFS, and OS were similar between thalidomide-resistant and thalidomide-sensitive patients. ${ }^{37}$ A retrospective analysis performed in The Netherlands further showed that response rate was not influenced by previous thalidomide or bortezomib treatment. ${ }^{38}$

In the MM-009 study, the response ( $\geq P R$ ) to lenalidomidedexamethasone was $68 \%$ in bortezomib exposed and $60 \%$ in bortezomib naïve patients. ${ }^{26}$ Results from the VISTA study also showed that lenalidomide-based therapy is equally effective in patients with or without previous bortezomib treatment. ${ }^{39}$ In contrast, a French study highlighted that previous bortezomib exposure was associated with significantly shorter PFS and OS in patients treated with lenalidomide-dexamethasone when compared to patients with no earlier bortezomib treatment. However, patients who received bortezomib had a median of two additional lines of therapy, compared to patients who did not receive bortezomib. ${ }^{36}$ Some other studies also suggest that prior bortezomib treatment is associated with lower efficacy of lenalidomide-dexamethasone. ${ }^{40-42}$ A retrospective single-center study showed that use of both thalidomide and bortezomib prior to lenalidomide-dexamethasone was associated with a significant reduction in TTP and OS. ${ }^{41}$

\section{Regimens with lenalidomide and conventional cytotoxic agents}

Various other lenalidomide-based regimens have been studied to further improve the outcome of patients with relapsed/ refractory MM (Table 2). Lenalidomide in combination with adriamycin and dexamethasone (RAD) in refractory and relapsed $\mathrm{MM}$ resulted in a high response rate of $73 \%$ ( $\geq \mathrm{PR}$ ) including 15\% CR and 45\% VGPR, with hematologic toxicity and infections as the primary side effects. ${ }^{43}$ Another lenalidomide and chemotherapy combination tested in the setting of relapsed/refractory MM was lenalidomide combined with pegylated liposomal doxorubicin, vincristine, and dexamethasone with an overall response rate ( $\geq \mathrm{PR})$ of $75 \%$ including $29 \%$ CR or near CR. ${ }^{44}$ Myelosuppression, neuropathy, muscle cramps, and rash were the most common adverse events. Median PFS was 12 months, and median OS had not been reached at the time of publication.

Preliminary results from a phase I/II study show that the combination of lenalidomide with bendamustine and dexamethasone is effective and well tolerated. ${ }^{45}$ Responses were also achieved in patients with prior exposure to lenalidomide. A variation on this regimen, bendamustine-lenalidomide plus prednisone followed by lenalidomide maintenance was evaluated in another Phase I trial. Preliminary results show that this combination was well tolerated and active in the setting of relapsed/refractory disease. ${ }^{46}$ 


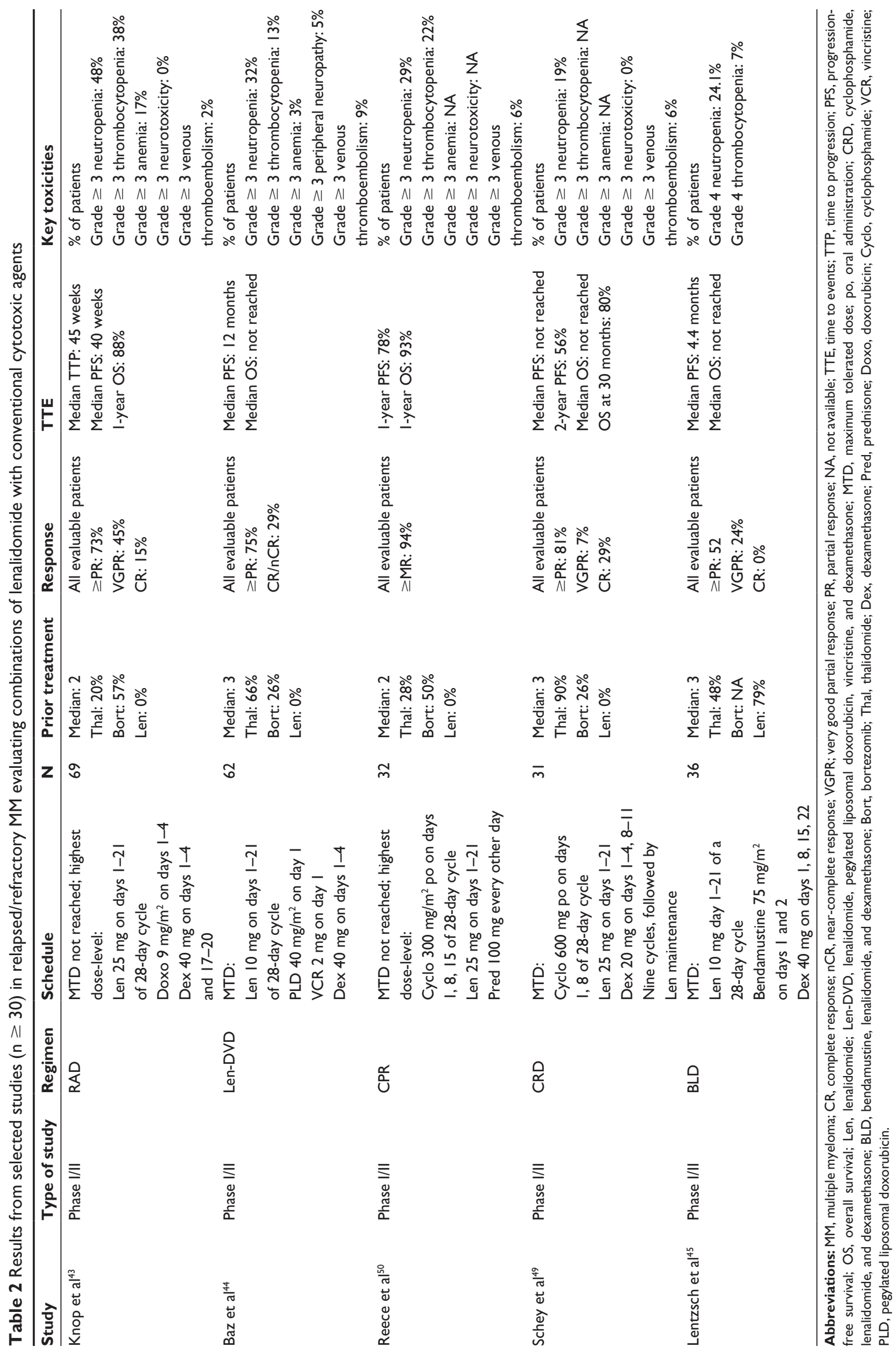


A retrospective study showed that the fully oral combination of lenalidomide $(10 \mathrm{mg})$ with continuous low-dose cyclophosphamide (Endoxan, Baxter $100 \mathrm{mg}$ orally [po]) and prednisone (REP) had a remarkably high activity with good tolerability in lenalidomide/dexamethasone-refractory MM ( $\geq$ minimal response (MR): 64\%; median PFS: 12.2 months). ${ }^{47}$ The efficacy of cyclophosphamide combined with lenalidomide and corticosteroids has also been described by Morgan et al. ${ }^{48}$ In a retrospective analysis, they treated relapsed lenalidomide-naïve MM patients with the combination of lenalidomide ( $25 \mathrm{mg} ; 21$ days, followed by 1 week rest), cyclophosphamide (500 mg po; days $1,8,15,21)$, and dexamethasone (40 mg po; days $1-4$ and $12-15$ of a 28 -day cycle; RCD regimen) resulting in $\geq \mathrm{MR}$ in $75 \%$ of patients. ${ }^{48}$ However, progression-free survival of 5.7 months seems inferior to REP (12.2 months). The prolonged progressionfree survival of patients treated with the REP regimen may be attributed to the continuous exposure of tumor cells to antimyeloma agents in this regimen, which possibly prevents the emergence of resistant clones.

Based on the promising results from these retrospective studies, a prospective Phase I/II study of cyclophosphamide, lenalidomide, and dexamethasone was performed in relapsed/ refractory lenalidomide-naïve MM with a median of three previous lines of therapy. The MTD was established at lenalidomide $25 \mathrm{mg}$ on days 1-21, cyclophosphamide $600 \mathrm{mg}$ on days 1 and 8 , and dexamethasone $20 \mathrm{mg}$ on days $1-4$ and 8-11 of each 28-day cycle. Hematological toxicity could be easily managed by dose-reductions. Of all 31 evaluable patients, 81\% achieved at least PR, including 29\% CR. ${ }^{49}$ The PFS at 2 years was $56 \%$, and the OS at 30 months was $80 \%{ }^{49}$ Another Phase I/II study is currently evaluating the combination of lenalidomide plus prednisone and cyclophosphamide, and has enrolled 32 lenalidomide-naïve patients. ${ }^{50}$ Preliminary data show good tolerability with high efficacy.

\section{Regimens with lenalidomide plus proteasome inhibitor}

In vitro studies demonstrate that lenalidomide sensitizes MM cells to bortezomib-induced apoptosis, which provided the rationale for clinical studies evaluating this combination (Table 3). ${ }^{6}$ A Phase I dose-escalation trial evaluated lenalidomide plus bortezomib in relapsed and refractory MM (median of five prior lines of therapy, including $87 \%$ of the patients with prior thalidomide, $55 \%$ with prior bortezomib, and 18\% prior lenalidomide). Dexamethasone was added if the patient experienced progression after the second cycle. The MTD was lenalidomide $15 \mathrm{mg}$ (days 1-14 of a 21-day cycle) and bortezomib $1.0 \mathrm{mg} / \mathrm{m}^{2}$ (days 1,4 , $8,11) .{ }^{51} \mathrm{MR}$ or better was observed in $61 \%$ of the patients, which included $39 \% \geq \mathrm{PR}$ and $8 \% \mathrm{CR}$ or near-CR. The most common treatment-related grade 3 to 4 toxicities included reversible neutropenia, thrombocytopenia, and anemia. ${ }^{51}$ This study was followed by a Phase II trial, which evaluated the efficacy of this combination at the MTD in 64 patients with relapsed or refractory disease. Preliminary results show an impressive overall response rate ( $\geq \mathrm{MR}$ ) of $78 \%$, including $64 \% \geq \mathrm{PR}$ and $25 \% \mathrm{CR}$ plus near-CR. ${ }^{52}$

Similar results were obtained in a Greek study, which demonstrated that the addition of bortezomib to lenalidomidedexamethasone was associated with a high response rate of at least PR in $63 \%$ of patients (median of two previous lines of therapy). ${ }^{53}$ Thalidomide-refractory disease was associated with an inferior response rate and survival. ${ }^{53}$ Another study evaluated lenalidomide, bortezomib, and dexamethasone followed by lenalidomide-dexamethasone maintenance as treatment of first relapse after autologous stem cell transplantation. Preliminary data showed a promising response rate ( $\geq$ PR: $71 \%$; $\geq$ VGPR: $43 \%$ ) with a low toxicity profile. ${ }^{54}$

The addition of pegylated doxorubicin to a modified schedule of lenalidomide-bortezomib-dexamethasone was evaluated in a Phase II study in relapsed/refractory MM. At least PR was achieved in $10(56 \%)$ of 18 patients (median of four lines of prior therapy). ${ }^{55}$

A dose-escalation study is investigating lenalidomide plus carfilzomib and dexamethasone in MM patients who have received a median of three previous therapies. Preliminary results show a response rate ( $\geq \mathrm{PR}$ ) of $55 \%$ across all cohorts. ${ }^{56}$ Responses were also observed in patients who had prior therapy with bortezomib or lenalidomide.

Lenalidomide-dexamethasone in combination with MLN9708, an oral, potent, reversible proteasome inhibitor, is currently being evaluated in newly diagnosed MM (NCT01217957 and NCT01383928). In addition, a phase III trial of weekly MLN9708, lenalidomide, and dexamethasone in patients with relapsed/refractory MM will soon be initiated (NCT01564537).

\section{Regimens with lenalidomide and thalidomide}

Based on a minimal overlapping side effect profile and different mechanisms of action between thalidomide (more antiangiogenic activity) and lenalidomide (more potent antiproliferative, cytotoxic, and immunomodulatory effects) in preclinical studies, the four-drug combination of lenalidomide, melphalan, prednisone, and thalidomide 


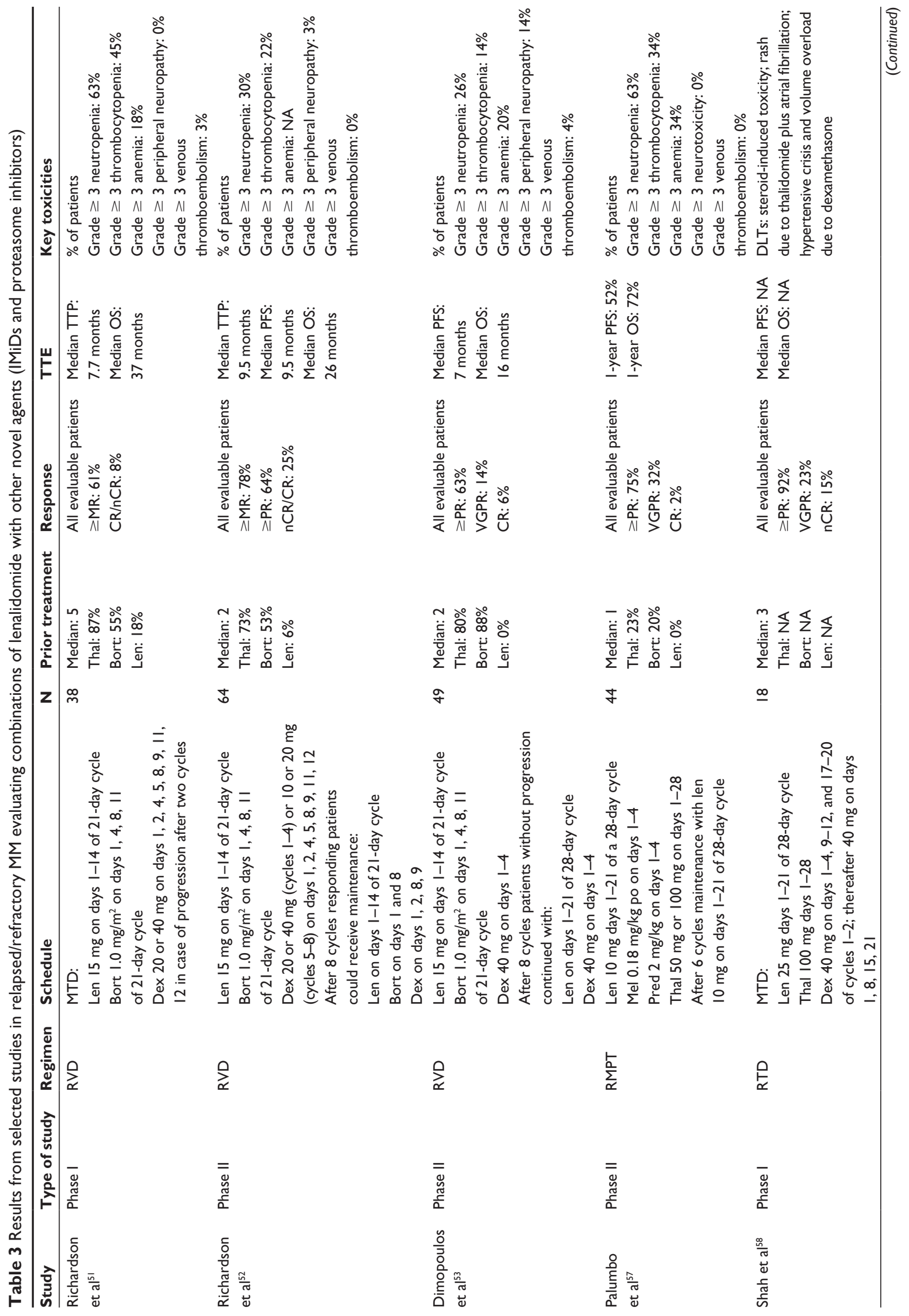




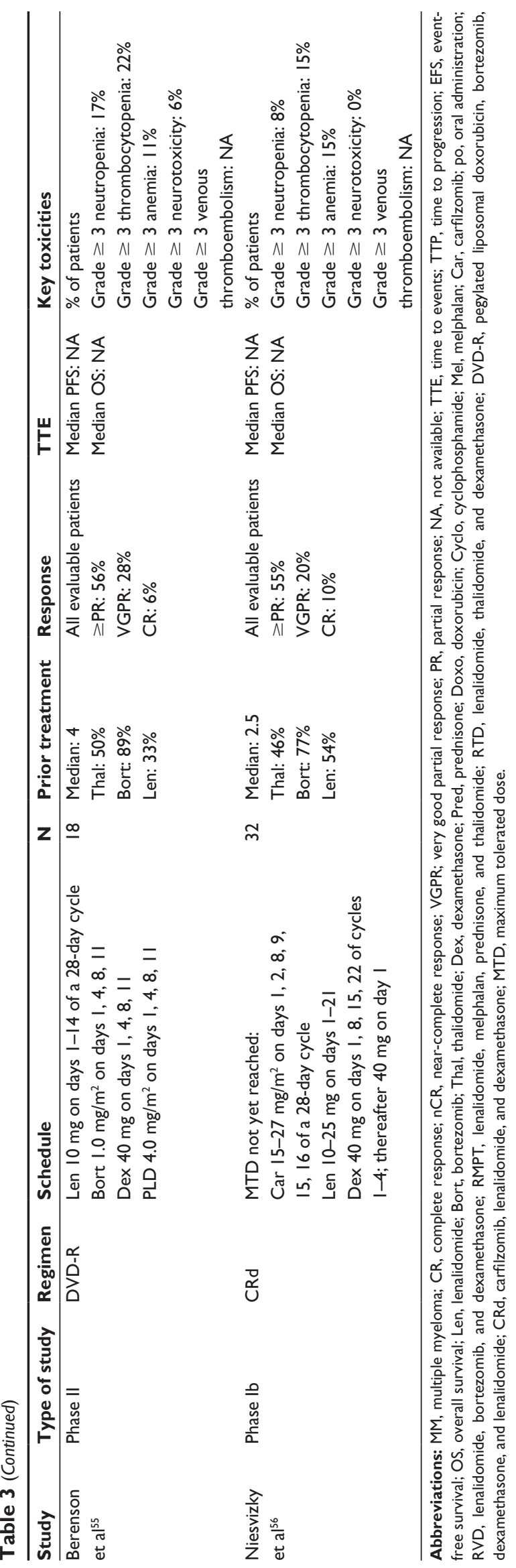

was tested in refractory/relapsed lenalidomide-naïve $\mathrm{MM}$ (Table 3). ${ }^{57} \mathrm{~A}$ high response rate was observed, with $75 \%$ of the 44 patients achieving at least a PR, including 32\% VGPR and $2 \% \mathrm{CR}$. Combining thalidomide with lenalidomide seems to increase the hematologic toxicity when compared to lenalidomide alone.

Another combination of both IMiDs is lenalidomide, thalidomide, and dexamethasone ${ }^{58}$ (Table 3). This combination was active, with at least PR in 12 out of 13 (92\%) evaluable patients (median of three prior lines of therapy). Interestingly, seven of eight patients (88\%) with lenalidomide-refractory disease achieved at least a PR, suggesting that thalidomide may modulate lenalidomide resistance.

\section{Regimens with lenalidomide and new novel agents mTor inhibitors}

In vitro studies demonstrating synergistic antimyeloma activity between lenalidomide and mTor inhibitors ${ }^{59}$ provided the rationale for testing the combination of lenalidomide and temsirolimus in a Phase I trial in relapsed/refractory MM patients $(\mathrm{n}=21$, median of three previous lines of therapy including 19\% lenalidomide). The maximum tolerated dose was lenalidomide $25 \mathrm{mg}$ on days 1-21 of a 28-day cycle with $15 \mathrm{mg}$ of temsirolimus weekly. Most common adverse events included fatigue, neutropenia, anemia, rash, and electrolyte abnormalities. The combination had modest activity, with PR in only two patients, and stable disease in 15 patients. ${ }^{60}$

Another Phase I study evaluated RAD001 and lenalidomide in relapsed/refractory MM (28 patients with a median of four previous therapies, including $50 \%$ prior lenalidomide). ${ }^{61}$ At least PR was obtained in $11 \%$ of patients.

\section{Histone deacetylase inhibitors}

A Phase I trial is evaluating vorinostat, lenalidomide plus dexamethasone in patients with relapsed/refractory MM. ${ }^{62}$ Patients had received a median of four prior therapies including lenalidomide in $45 \%$, thalidomide in $71 \%$, and bortezomib in $65 \%$ cases. Fatigue, cytopenias, and diarrhea were the most common adverse events. At least PR was achieved in $53 \%$ of patients, including those previously exposed to lenalidomide. Median TTP was 5 months.

The combination of vorinostat, lenalidomide, and dexamethasone as a salvage therapy was also evaluated in 29 lenalidomide-dexamethasone-refractory MM patients with a median of four previous lines of therapy. ${ }^{63}$ At least PR 
was achieved in $24 \%$ of patients, with a median duration of response of 4 months. Common toxicities included fatigue, myelosuppression, and diarrhea.

\section{Akt inhibitor}

Perifosine, an oral Akt inhibitor, was combined with lenalidomide and dexamethasone in a Phase I trial. ${ }^{64}$ Patients had received a median of two prior lines of treatment, and patients refractory to lenalidomide plus dexamethasone were excluded. A total of $50 \%$ of the patients achieved at least PR, and median PFS was 10.8 months.

\section{Lenalidomide and monoclonal antibody therapy}

The ability of lenalidomide to activate multiple arms of the patient's immune system including enhanced antibodydependent cell-mediated cytotoxicity, ${ }^{65,66}$ coupled with its ability to modulate important signaling cascades in MM cells, forms the rationale to combine lenalidomide with monoclonal antibodies. ${ }^{67}$ Preliminary results from ongoing trials show encouraging results with acceptable toxicity for lenalidomide combined with elotuzumab (anti-CS1 antibody), ${ }^{68,69}$ lorvotuzumab mertansine (anti-CD56 antibody conjugated to DM1), ${ }^{70}$ and dacetuzumab (anti-CD40 antibody). ${ }^{71}$

In vitro studies also demonstrated enhanced cytotoxicity of NK cells against MM cells of the combination of NK cell activating antibodies and lenalidomide, which also activates NK cells. ${ }^{72}$ Based on these data, a Phase I/II trial is currently evaluating lenalidomide combined with 1-7F9, a fully human anti-KIR antibody, in relapsed/refractory MM. ${ }^{72}$

\section{Lenalidomide and cancer vaccines}

Suppression of cytotoxic T cells by cytokines such as TGF$\beta$, recruitment of regulatory $\mathrm{T}$ cells, and altered expression of immune suppressor molecules on MM cells or immune effector cells, contributes to immune evasion in MM. In addition, the function of dendritic cells and NK cells is severely impaired in MM. In a mouse model, a lymphoma vaccine in combination with lenalidomide improved survival when compared to vaccine or lenalidomide alone. Lenalidomide treatment was accompanied with enhanced cellular immunity and ameliorated immune suppression. ${ }^{73}$ In vitro studies showed that lenalidomide also enhanced $\mathrm{T}$ cell activation in response to stimulation by a dendritic cell/MM fusion vaccine. ${ }^{74} \mathrm{~A}$ recent clinical study in MM patients showed that lenalidomide augmented humoral and cellular responses to the polyvalent pneumococcal vaccine, Prevnar (Wyeth
Pharmaceuticals, Inc, Madison, NJ; Pfizer Inc, New York, NY). ${ }^{75}$ Altogether, these studies indicate that lenalidomide has the potential to improve immune dysfunction and can serve as an adjuvant for MM vaccines.

Increasing evidence also suggests that IMiDs enhance the graft-versus-myeloma effect mediated by donor $\mathrm{T}$ cells or donor NK cells after allogeneic stem cell transplantation or donor lymphocyte infusions. ${ }^{76-80}$ Unfortunately, use of IMiDs in this setting seems also to be associated with increased occurrence of graft-versus-host disease. ${ }^{78,79}$

\section{Cytogenetics}

Data from a Canadian study in relapsed or refractory MM suggest that lenalidomide-dexamethasone can overcome the poor prognosis conferred by $\operatorname{del}(13 q)$ and $t(4 ; 14)$, but not $\operatorname{del}(17 \mathrm{p})$ (all detected by fluorescence in situ hybridization). ${ }^{40}$ However, results from a French multicenter study testing lenalidomide-dexamethasone in the relapsed setting showed an inferior PFS in patients with del(13q) and $t(4 ; 14) .{ }^{36}$ Also, del(1p21) adversely affected the outcome of patients treated with lenalidomide-dexamethasone. ${ }^{42}$

In other lenalidomide-based combinations, $\operatorname{del}(17 \mathrm{p})$ remains a negative prognostic factor, with some combinations overcoming poor prognosis of both $t(4 ; 14)$ and $\operatorname{del}(13 q)$. Response and TTP were identical in patients with or without $\operatorname{del}(13 q)$ or $\mathrm{t}(4 ; 14)$ following RAD treatment, but del $(17 p)$ remained associated with an inferior response rate and shortened TTP. ${ }^{43}$ In addition, when bortezomib was added to lenalidomide-dexamethasone, del(17p) was still associated with an inferior response rate and survival. But bortezomib added to lenalidomide-dexamethasone overcame part of the adverse impact conferred by del(13q), ampl(1q21), and $t(4 ; 14) .{ }^{53}$ The PFS of patients treated with lenalidomide, melphalan, prednisone and thalidomide was independent of the presence of $\operatorname{del}(13 q)$, but was inferior in patients with $\mathrm{t}(4 ; 14) .{ }^{57}$

\section{Lenalidomide treatment in frail or elderly patients with relapsed/ refractory MM}

More than half of all new cases of MM occur in patients 65 years of age or older, ${ }^{81}$ whereas the proportion of elderly relapsed/refractory MM patients enrolled in clinical trials decreases with age, and no specific trials are currently available for unfit elderly MM patients. Furthermore, during the last decade, the improvement in survival was more pronounced in younger patients, ${ }^{81}$ which is likely due to patient characteristics such as lower performance status and comorbidities in 
the elderly which strongly impact chemotherapy feasibility and tolerance. In addition, biologic differences between tumors may play a role. This highlights the need for further treatment innovations in this population.

Dose adjustments of lenalidomide and other components of the salvage regimen, such as dexamethasone, are needed to keep patients on therapy and prevent treatment discontinuation. Depending on the number of risk factors (such as age $\geq 75$ years, frailty, and comorbidities), the starting dose of lenalidomide should be 25,15 , or $10 \mathrm{mg}$ on days $1-21$, and the starting dose of dexamethasone should be $40 \mathrm{mg}, 20 \mathrm{mg}$, or $10 \mathrm{mg}$ weekly. ${ }^{82,83}$ A Phase II trial in relapsed/refractory $\mathrm{MM}$ patients aged $\geq 60$ years and/or with a creatinine clearance $<60 \mathrm{~mL} / \mathrm{min}$ showed that lower doses of lenalidomide (15 mg) and dexamethasone reduced the incidence of hematological toxicities, infections, and VTE without compromising efficacy when compared to standard dose lenalidomide-dexamethasone (MM-010 and MM-009). ${ }^{84}$ Another lenalidomide-containing salvage regimen with proven efficacy accompanied with mild toxicity, which may be beneficial to frail MM patients, includes dose-adjusted lenalidomide-cyclophosphamide-prednisone. ${ }^{47}$ Careful monitoring of toxicity and prompt administration of supportive care such as G-CSF in case of neutropenia is important in this group of patients.

\section{Renal impairment}

Bortezomib clearance is independent of renal function and overcomes the adverse impact of renal dysfunction. Importantly, it also improves renal function to a higher degree than conventional chemotherapy or IMiD-based regimens. This suggests that relapsed/refractory patients presenting with renal insufficiency should receive bortezomib-based treatment. However, in case of bortezomib-refractory disease or intolerance to bortezomib, thalidomide or dose-adjusted lenalidomide-based regimens can be considered. ${ }^{85}$

Lenalidomide is a renally metabolized drug, and without dose adjustments myelosuppression is more frequent in patients with renal impairment. ${ }^{86-88}$ In a subgroup analysis of MM-009 and MM-010 studies (starting dose of lenalidomide $25 \mathrm{mg}$ for all patients), response and TTP was independent of renal function. Improvement of renal function was observed in the majority of patients with renal impairment. However, patients with severe renal impairment had a shorter OS. ${ }^{87}$

With dosing of lenalidomide being administered according to creatinine clearance (Table 4), toxicity of lenalidomide
Table 4 Adjustment of lenalidomide dose in case of renal impairment

\begin{tabular}{ll}
\hline Creatinine clearance & Lenalidomide (days I-2I of a 28-day cycle) \\
\hline$\geq 50 \mathrm{~mL} / \mathrm{min}$ & $25 \mathrm{mg} /$ day (standard dose) \\
$30-50 \mathrm{~mL} / \mathrm{min}$ & $10 \mathrm{mg} /$ day \\
$\leq 30 \mathrm{~mL} / \mathrm{min}$ & $15 \mathrm{mg} / 2$ days \\
Dialysis & $5 \mathrm{mg} /$ day; on days of dialysis, dose should be \\
& administered after dialysis \\
\hline
\end{tabular}

was independent of renal function. ${ }^{89}$ The response rate, PFS, and OS following lenalidomide-dexamethasone were identical between patients with and without renal impairment, and treatment was associated with improvement of renal function. However, a retrospective single center study from Germany with a starting dose of lenalidomide according to renal function showed that TTP following lenalidomidedexamethasone was significantly shorter in the case of severe renal impairment, probably due to dose interruptions and reductions resulting from toxicity. ${ }^{41}$

Adjusted dose lenalidomide-based therapy can also be administered to patients requiring dialysis, which is effective, but accompanied by a high incidence of neutropenia and infections. ${ }^{90}$

\section{Management of adverse events associated with lenalidomide Neutropenia}

Neutropenia increases the risk of bacterial and fungal infection, and is a common adverse event of lenalidomide treatment. The incidence of grade 3/4 neutropenia in the MM-009 and MM-010 studies was $41.2 \%$ and 29.5\%, respectively, in lenalidomide plus dexamethasone-treated patients, whereas it was only $4.6 \%$ and $2.3 \%$, respectively, in the placebo plus dexamethasone group. ${ }^{25,26}$ The risk of developing grade 3/4 neutropenia is higher when lenalidomide is combined with other chemotherapeutic agents such as alkylating drugs (eg, MPR) or doxorubicin (eg, RAD). Extensive previous treatment is also an important risk factor. Therapy-related toxicities including infections may lead to early treatment discontinuations, thereby negatively affecting outcomes. ${ }^{91}$ When patients start with lenalidomide-based treatment, the blood counts should be monitored biweekly, but in case of pre-existing cytopenia, weekly monitoring is recommended. Growth factor support, and sometimes dose adjustments and dose interruptions, should be considered in case neutropenia develops (Table 5). Patients at high risk of developing neutropenia 
Table 5 Supportive care for the management or prevention of adverse events associated with lenalidomide

\begin{tabular}{ll}
\hline Averse event & Supportive care \\
\hline Neutropenia & G-CSF \\
Anemia & Red cell transfusion; start of erythropoietin \\
Thrombocytopenia & Platelet transfusion \\
Diarrhea & Loperamide \\
Rash & Limited, localized rash: antihistamines \\
& and topical steroids \\
& Mild but extensive rash: short course \\
& of low-dose prednisone \\
& Thromboprophylaxis (aspirin for patients at \\
VTE & standard risk for VTE and LMWH or adjusted- \\
& dose warfarin in high-risk patients) is indicated \\
& when lenalidomide is combined with \\
& dexamethasone or cytotoxic agents \\
\hline
\end{tabular}

Note: ${ }^{\dagger}$ Concomitant use of erythropoietin with lenalidomide-based combinations may increase risk of VTE.

Abbreviations: G-CSF, granulocyte-colony stimulating factor; VTE, venous thromboembolism; LMWH, low molecular weight heparin.

based on patient-, MM-, and treatment-related factors may benefit from primary prophylaxis with G-CSF.

\section{Venous thromboembolism}

Lenalidomide used as a single agent does not increase the risk of VTE. However, treatment with lenalidomide plus dexamethasone or cytotoxic agents results in a higher incidence of VTE..$^{25,26,48}$ In the MM-009 and MM-010 trials, the incidence of grade 3 and 4 thromboembolic events was $11.4 \%$ and $14.7 \%$, respectively, in the lenalidomide plus dexamethasone group, which was significantly higher compared to $4.6 \%$ and $3.4 \%$, respectively, in the placebo plus dexamethasone group. ${ }^{25,26}$ Importantly, in both studies thromboprophylaxis was not required. Risk factors for thromboembolic events associated with lenalidomide-dexamethasone treatment include high-dose dexamethasone ${ }^{32,92-94}$ and concomitant erythropoietin administration. ${ }^{92,94-96}$ A subgroup analysis of the MM-009 and MM-010 trials showed an increased rate of VTE during lenalidomide-dexamethasone therapy in previously thalidomide-exposed patients when compared with thalidomide-naïve patients. ${ }^{35}$ Interestingly, data suggest that the frequency of VTE may be markedly reduced when bortezomib is combined with IMiD-based regimens with high thrombogenic potential, even when no thromboprophylaxis was administered. ${ }^{51,97-103}$

In addition, patient-related factors such as advanced age, history of VTE, immobilization, inherited thrombophilic disorders, and comorbidities such as diabetes mellitus and cardiac disease, contribute to the development of VTE during lenalidomide therapy. Patients treated with lenalidomidedexamethasone who developed a VTE did not experience shorter OS or TTP. ${ }^{104}$

Importantly, with thalidomide and lenalidomide-based combination therapies, prophylactic treatment with aspirin in patients at standard risk for VTE and low molecular weight heparin, or adjusted dose warfarin for high-risk patients reduces the frequency of VTE (Table 5). ${ }^{93,95,96,105,106}$

\section{Secondary malignancies}

There have been recent concerns over the use of lenalidomide and the risk of developing second primary malignancies. There is an increased incidence of second primary malignancies in newly diagnosed MM patients receiving lenalidomide plus melphalan/prednisone (MPR). ${ }^{107,108}$ In the randomized Phase III, MM-015 study, the 3 -year rate of invasive primary tumors was $7 \%$ in patients treated with MPR, 7\% in patients treated with MPR followed by lenalidomide maintenance (MPR-R), but only $3 \%$ in the melphalan/prednisone group. ${ }^{108}$ In addition, patients receiving lenalidomide maintenance following high-dose therapy with autologous stem cell rescue had a significantly higher incidence of second primary cancers. ${ }^{109-112}$ In the Intergroupe Francophone du Myelome trial, the incidence of second primary cancers was 3.1 per 100 patient-years in the lenalidomide group versus 1.2 per 100 patient-years in the placebo group. ${ }^{111}$ In the CALGB study, $3.5 \%$ and $4.3 \%$ of the patients in the lenalidomide maintenance group developed new hematologic cancers and solid-tumor cancers (excluding nonmelanoma skin cancers), respectively. ${ }^{12}$ The corresponding numbers are $0.4 \%$ and $2.2 \%$ in the placebo group. ${ }^{112}$

A retrospective pooled analysis of 11 clinical trials of lenalidomide-based therapy has addressed this issue in the relapsed/refractory setting, ${ }^{113}$ however, in the absence of prospective studies, conclusions regarding the incidence of second primary cancers are more difficult to draw. In a pooled analysis of 3846 relapsed/refractory MM patients treated with lenalidomide as a single agent $(7 \%)$ or in combination with dexamethasone (93\%), the incidence rate (events per 100 patient-years) of invasive second primary malignancies was 2.08 , which is comparable to that expected according to the Surveillance, Epidemiology and End Results cancer registry (2.1). ${ }^{113}$ The incidence rates (events per 100 patient-years) of second primary malignancies, excluding noninvasive skin cancers, in the MM-009 and MM-010 trials was 1.71 for lenalidomide plus dexamethasone, and 0.91 for placebo plus 
dexamethasone. This difference was not statistically significant, and it was also consistent with the expected incidence of invasive cancer in the general population. ${ }^{113}$ However, there was an increased occurrence of noninvasive skin cancers in the lenalidomide plus dexamethasone group compared to the dexamethasone only group (incidence rate: 2.40 vs 0.91 ). Although there is an increased incidence of non-invasive skin cancers in this patient group, there remains a positive risk-benefit profile of lenalidomide plus dexamethasone in relapsed/refractory MM. ${ }^{113}$

\section{Other adverse events}

Other common adverse events associated with lenalidomide treatment include thrombocytopenia, anemia, rash, and diarrhea. These toxicities can be managed with dose reductions or interruptions, as well as with the start of appropriate supportive care (Table 5). ${ }^{114}$

\section{Concluding remarks}

Lenalidomide plus dexamethasone and other lenalidomidebased combinations are effective treatment options for relapsed/refractory MM patients. Several studies demonstrate that continued treatment with lenalidomide is associated with improved survival. Appropriate dose adjustments and institution of supportive care are therefore very important to enable patients to continue treatment with lenalidomidebased therapies until disease progression.

The introduction of the novel agents, thalidomide, lenalidomide, and bortezomib, ${ }^{115}$ and the application of high-dose therapy with autologous stem cell rescue have improved the survival of MM patients. However, event-free and overall survival for patients that are refractory to both an IMiD and bortezomib is only 5 and 9 months, respectively, ${ }^{116}$ indicating that new drugs are still needed for continued disease control. Novel agents are currently being evaluated in clinical trials, and include second generation IMiDs such as pomalidomide, and second generation proteasome inhibitors, such as carfilzomib, MLN 9708, and marizomib. Drugs belonging to other classes, such as histone deacetylase inhibitors, Akt inhibitors, mTor inhibitors, and several monoclonal antibodies including elotuzumab and daratumumab, hold promise for improving the outcome of patients with lenalidomide and bortezomib refractory disease. ${ }^{67,117}$ Incorporation of biomarker assessment (using techniques such as fluorescence in situ hybridization, gene expression profiling, array based comparative genomic hybridization, single nucleotide polymorphism array, microRNA array, or high throughput sequencing) in future studies in relapsed/refractory MM will help to evaluate the risk/benefit profile and tailor individualized therapeutic approaches. Altogether, this will hopefully translate to further improvement in outcomes for MM patients.

\section{Acknowledgment}

NWCJvdD was supported by the Dutch Cancer Society.

\section{Conflict of interest disclosure}

NWCJvdD has received research support from Celgene, and honoraria as a speaking fee from Johnson and Johnson and Celgene. CSM has received in the past honoraria from Millennium Pharmaceuticals, Novartis Pharmaceuticals, Bristol-Myers Squibb, Merck and Co, Celgene, licensing royalties from PharmaMar, and research funding from Amgen Pharmaceuticals, AVEO Pharma, EMD Serono, Sunesis, and Johnson and Johnson. HML has received research support from Celgene, Genmab, and Ortho-Biotech. PGR has served on the advisory board of Millennium Pharmaceuticals, Celgene Corporation, Novartis, Johnson and Johnson, and Bristol Myers Squibb. KCA is the scientific founder of Acetylon, and is on the advisory boards of Celgene, Millennium, Onyx, BMS, and Merck. GG, RWJG, JJ, TH, JPL, ISN, and RAR declare no competing financial interests.

\section{References}

1. Kotla V, Goel S, Nischal S, et al. Mechanism of action of lenalidomide in hematological malignancies. J Hematol Oncol. 2009;2:36.

2. Vallet S, Witzens-Harig M, Jaeger D, Podar K. Update on immunomodulatory drugs (IMiDs) in hematologic and solid malignancies. Expert Opin Pharmacother. 2012;13(4):473-494.

3. Davies F, Baz R. Lenalidomide mode of action: linking bench and clinical findings. Blood Rev. 2010;24 Suppl 1:S13-S19.

4. Richardson PG, Schlossman RL, Weller E, et al. Immunomodulatory drug CC-5013 overcomes drug resistance and is well tolerated in patients with relapsed multiple myeloma. Blood. 2002;100(9):3063-3067.

5. Hideshima T, Chauhan D, Shima Y, et al. Thalidomide and its analogs overcome drug resistance of human multiple myeloma cells to conventional therapy. Blood. 2000;96(9):2943-2950.

6. Mitsiades N, Mitsiades CS, Poulaki V, et al. Apoptotic signaling induced by immunomodulatory thalidomide analogs in human multiple myeloma cells: therapeutic implications. Blood. 2002;99(12):4525-4530.

7. Verhelle D, Corral LG, Wong K, et al. Lenalidomide and CC-4047 inhibit the proliferation of malignant $\mathrm{B}$ cells while expanding normal CD34+ progenitor cells. Cancer Res. 2007;67(2):746-755.

8. Jakubikova J, Adamia S, Kost-Alimova M, et al. Lenalidomide targets clonogenic side population in multiple myeloma: pathophysiologic and clinical implications. Blood. 2011;117(17):4409-4419.

9. Ito $\mathrm{T}$, Ando $\mathrm{H}$, Suzuki T, et al. Identification of a primary target of thalidomide teratogenicity. Science. 2010;327(5971):1345-1350.

10. Zhu YX, Braggio E, Shi CX, et al. Cereblon expression is required for the antimyeloma activity of lenalidomide and pomalidomide. Blood. 2011;118(18):4771-4779.

11. Geitz H, Handt S, Zwingenberger K. Thalidomide selectively modulates the density of cell surface molecules involved in the adhesion cascade. Immunopharmacology. 1996;31(2-3):213-221. 
12. Hideshima T, Chauhan D, Schlossman R, Richardson P, Anderson KC. The role of tumor necrosis factor alpha in the pathophysiology of human multiple myeloma: therapeutic applications. Oncogene. 2001;20(33):4519-4527.

13. Corral LG, Haslett PA, Muller GW, et al. Differential cytokine modulation and $\mathrm{T}$ cell activation by two distinct classes of thalidomide analogues that are potent inhibitors of TNF-alpha. J Immunol. 1999;163(1): 380-386.

14. Gupta D, Treon SP, Shima Y, et al. Adherence of multiple myeloma cells to bone marrow stromal cells upregulates vascular endothelial growth factor secretion: therapeutic applications. Leukemia. 2001;15(12): 1950-1961.

15. De Luisi A, Ferrucci A, Coluccia AM, et al. Lenalidomide restrains motility and overangiogenic potential of bone marrow endothelial cells in patients with active multiple myeloma. Clin Cancer Res. 2011;17(7): 1935-1946.

16. Breitkreutz I, Raab MS, Vallet S, et al. Lenalidomide inhibits osteoclastogenesis, survival factors and bone-remodeling markers in multiple myeloma. Leukemia. 2008;22(10):1925-1932.

17. Davies FE, Raje N, Hideshima T, et al. Thalidomide and immunomodulatory derivatives augment natural killer cell cytotoxicity in multiple myeloma. Blood. 2001;98(1):210-216.

18. Chang DH, Liu N, Klimek V, et al. Enhancement of ligand-dependent activation of human natural killer T cells by lenalidomide: therapeutic implications. Blood. 2006;108(2):618-621.

19. Galustian C, Meyer B, Labarthe MC, et al. The anti-cancer agents lenalidomide and pomalidomide inhibit the proliferation and function of T regulatory cells. Cancer Immunol Immunother. 2009;58(7): 1033-1045.

20. Muller GW, Chen R, Huang SY, et al. Amino-substituted thalidomide analogs: potent inhibitors of TNF-alpha production. Bioorg Med Chem Lett. 1999;9(11):1625-1630.

21. LeBlanc R, Hideshima T, Catley LP, et al. Immunomodulatory drug costimulates T cells via the B7-CD28 pathway. Blood. 2004;103(5): 1787-1790.

22. Gorgun G, Calabrese E, Soydan E, et al. Immunomodulatory effects of lenalidomide and pomalidomide on interaction of tumor and bone marrow accessory cells in multiple myeloma. Blood. 2010;116(17):3227-3237.

23. Richardson PG, Blood E, Mitsiades CS, et al. A randomized phase 2 study of lenalidomide therapy for patients with relapsed or relapsed and refractory multiple myeloma. Blood. 2006;108(10): 3458-3464.

24. Richardson P, Jagannath S, Hussein M, et al. Safety and efficacy of single-agent lenalidomide in patients with relapsed and refractory multiple myeloma. Blood. 2009;114(4):772-778.

25. Dimopoulos M, Spencer A, Attal M, et al; for Multiple Myeloma (010) Study Investigators. Lenalidomide plus dexamethasone for relapsed or refractory multiple myeloma. N Engl J Med. 2007;357(21) 2123-2132.

26. Weber DM, Chen C, Niesvizky R, et al; for Multiple Myeloma (009) Study Investigators. Lenalidomide plus dexamethasone for relapsed multiple myeloma in North America. $N$ Engl J Med. 2007;357(21): 2133-2142.

27. Dimopoulos MA, Chen C, Spencer A, et al. Long-term follow-up on overall survival from the MM-009 and MM-010 phase III trials of lenalidomide plus dexamethasone in patients with relapsed or refractory multiple myeloma. Leukemia. 2009;23(11):2147-2152.

28. Harousseau JL, Dimopoulos MA, Wang M, et al. Better quality of response to lenalidomide plus dexamethasone is associated with improved clinical outcomes in patients with relapsed or refractory multiple myeloma. Haematologica. 2010;95(10):1738-1744.

29. San-Miguel JF, Dimopoulos MA, Stadtmauer EA, et al. Effects of lenalidomide and dexamethasone treatment duration on survival in patients with relapsed or refractory multiple myeloma treated with lenalidomide and dexamethasone. Clin Lymphoma Myeloma Leuk. 2011;11(1):38-43.
30. San Miguel J, Dimopoulos M, Bravo ML, Weber D. Longer duration of treatment and maintenance of best response with lenalidomide + dexamethasone increases overall survival (OS) in patients with relapsed/refractory multiple myeloma [abstract]. Haematologica. 2009;94(Suppl 2):382.

31. San Miguel JF, Dimopoulos M, Weber D, et al. Dexamethasone dose adjustments seem to result in better efficacy and improved tolerability in patients with relapsed/refractory multiple myeloma who are treated with lenalidomide/dexamethasone (MM009/010 sub-analysis) [abstract]. Blood (ASH Annual Meeting Abstracts). 2007;110(11): 2712.

32. Rajkumar SV, Jacobus S, Callander NS, et al; for Eastern Cooperative Oncology Group. Lenalidomide plus high-dose dexamethasone versus lenalidomide plus low-dose dexamethasone as initial therapy for newly diagnosed multiple myeloma: an open-label randomised controlled trial. Lancet Oncol. 2010;11(1):29-37.

33. Dimopoulos MA, Hussein M, Swern AS, Weber D. Impact of lenalidomide dose on progression-free survival in patients with relapsed or refractory multiple myeloma. Leukemia. 2011;25(10): $1620-1626$

34. Stadtmauer EA, Weber DM, Niesvizky R, et al. Lenalidomide in combination with dexamethasone at first relapse in comparison with its use as later salvage therapy in relapsed or refractory multiple myeloma. Eur J Haematol. 2009;82(6):426-432.

35. Wang M, Dimopoulos MA, Chen C, et al. Lenalidomide plus dexamethasone is more effective than dexamethasone alone in patients with relapsed or refractory multiple myeloma regardless of prior thalidomide exposure. Blood. 2008;112(12):4445-4451.

36. Avet-Loiseau H, Soulier J, Fermand JP, et al; for IFM and MAG groups. Impact of high-risk cytogenetics and prior therapy on outcomes in patients with advanced relapsed or refractory multiple myeloma treated with lenalidomide plus dexamethasone. Leukemia. 2010;24(3):623-628.

37. Guglielmelli T, Bringhen S, Rrodhe S, et al. Previous thalidomide therapy may not affect lenalidomide response and outcome in relapse or refractory multiple myeloma patients. Eur J Cancer. 2011;47(6): 814-818.

38. Kneppers E, Lokhorst HM, Eeltink CM, et al. Analysis of efficacy and prognostic factors of lenalidomide treatment as part of a Dutch compassionate use program. Clin Lymphoma Myeloma Leuk. 2010;10(2): $138-143$.

39. Mateos MV, Richardson PG, Schlag R, et al. Bortezomib plus melphalan and prednisone compared with melphalan and prednisone in previously untreated multiple myeloma: updated follow-up and impact of subsequent therapy in the phase III VISTA trial. J Clin Oncol. 2010;28(13): 2259-2266.

40. Reece D, Song KW, Fu T, et al. Influence of cytogenetics in patients with relapsed or refractory multiple myeloma treated with lenalidomide plus dexamethasone: adverse effect of deletion 17p13. Blood. 2009;114(3):522-525.

41. Klein U, Neben K, Hielscher T, Heiss C, Ho AD, Goldschmidt H. Lenalidomide in combination with dexamethasone: effective regimen in patients with relapsed or refractory multiple myeloma complicated by renal impairment. Ann Hematol. 2011;90(4):429-439.

42. Chang H, Jiang A, Qi C, Trieu Y, Chen C, Reece D. Impact of genomic aberrations including chromosome 1 abnormalities on the outcome of patients with relapsed or refractory multiple myeloma treated with lenalidomide and dexamethasone. Leuk Lymphoma. 2010;51(11):2084-2091.

43. Knop S, Gerecke C, Liebisch P, et al. Lenalidomide, adriamycin, and dexamethasone (RAD) in patients with relapsed and refractory multiple myeloma: a report from the German Myeloma Study Group DSMM (Deutsche Studiengruppe Multiples Myelom). Blood. 2009;113(18): 4137-4143.

44. Baz R, Walker E, Karam MA, et al. Lenalidomide and pegylated liposomal doxorubicin-based chemotherapy for relapsed or refractory multiple myeloma: safety and efficacy. Ann Oncol. 2006;17(12):1766-1771. 
45. Lentzsch S, O’Sullivan A, Kennedy R, et al. Combination of bendamustine, lenalidomide, and dexamethasone (BLD) in patients with refractory or relapsed multiple myeloma is safe and highly effective: results of phase I/II open-label, dose escalation study [abstract]. Blood (ASH Annual Meeting Abstracts). 2011;118(21):304.

46. Ponisch W, Heyn S, Wagner I, et al. Combined bendamustine, prednisolone and lenalidomide (RBP) in refractory or relapsed multiple myeloma. First results of a phase I clinical trial [abstract]. Blood (ASH Annual Meeting Abstracts). 2010;116(21):1971.

47. van de Donk NW, Wittebol S, Minnema MC, Lokhorst HM. Lenalidomide (Revlimid) combined with continuous oral cyclophosphamide (endoxan) and prednisone (REP) is effective in lenalidomide/dexamethasone-refractory myeloma. Br J Haematol. 2010;148(2):335-337.

48. Morgan GJ, Schey SA, Wu P, et al. Lenalidomide (Revlimid), in combination with cyclophosphamide and dexamethasone (RCD), is an effective and tolerated regimen for myeloma patients. Br J Haematol. 2007;137(3):268-269.

49. Schey SA, Morgan GJ, Ramasamy K, et al. The addition of cyclophosphamide to lenalidomide and dexamethasone in multiply relapsed/ refractory myeloma patients; a phase I/II study. Br J Haematol. 2010; 150(3):326-333

50. Reece DE, Masih-Khan E, Khan A, et al. Phase I-II trial of oral cyclophosphamide, prednisone and lenalidomide $(\operatorname{Revlimid}(\mathrm{R}))$ (CPR) for the treatment of patients with relapsed and refractory multiple myeloma [abstract]. Blood (ASH Annual Meeting Abstracts). 2010;116(21):3055.

51. Richardson PG, Weller E, Jagannath S, et al. Multicenter, phase I, dose-escalation trial of lenalidomide plus bortezomib for relapsed and relapsed/refractory multiple myeloma. J Clin Oncol. 2009;27(34): 5713-5719.

52. Richardson PG, Jagannath S, Jakubowiak AJ, et al. Phase II trial of lenalidomide, bortezomib, and dexamethasone in patients (pts) with relapsed and relapsed/refractory multiple myeloma (MM): updated efficacy and safety data after $>2$ years of follow-up [abstract]. Blood (ASH Annual Meeting Abstracts). 2010;116(21):3049.

53. Dimopoulos MA, Kastritis E, Christoulas D, et al. Treatment of patients with relapsed/refractory multiple myeloma with lenalidomide and dexamethasone with or without bortezomib: prospective evaluation of the impact of cytogenetic abnormalities and of previous therapies. Leukemia. 2010;24(10):1769-1778.

54. Belhadj K, Amraoui K, Safar V, et al. Lenalidomide plus bortezomib and dexamethasone (RVD) followed by lenalidomide plus dexamethasone as salvage treatment for myeloma patients in first relapse after autologous stem cell transplantation. Single institution experience [abstract]. Blood (ASH Annual Meeting Abstracts). 2011;118(21):5143.

55. Berenson JR, Yellin O, Cartmell AD, et al. A phase II study of pegylated liposomal doxorubicin (PLD), bortezomib, dexamethasone and lenalidomide (DVD-R) for patients with relapsed/refractory (R/R) multiple myeloma (MM) [abstract]. Blood (ASH Annual Meeting Abstracts). 2010;116(21):3044.

56. Niesvizky R, Wang L, Orlowski RZ, et al. Phase Ib multicenter dose escalation study of carfilzomib plus lenalidomide and low dose dexamethasone (CRd) in relapsed and refractory multiple myeloma (MM) [abstract]. Blood (ASH Annual Meeting Abstracts). November 20, 2009;114(22):304.

57. Palumbo A, Larocca A, Falco P, et al. Lenalidomide, melphalan, prednisone and thalidomide (RMPT) for relapsed/refractory multiple myeloma. Leukemia. 2010;24(5):1037-1042.

58. Shah JJ, Orlowski RZ, Alexanian R, et al. Phase I trial of the combination of lenalidomide, thalidomide and dexamethasone in relapsed/ refractory multiple myeloma [abstract]. Blood (ASH Annual Meeting Abstracts). 2010;116(21):1948.

59. Raje N, Kumar S, Hideshima T, et al. Combination of the mTOR inhibitor rapamycin and CC-5013 has synergistic activity in multiple myeloma. Blood. 2004;104(13):4188-4193.
60. Hofmeister CC, Yang X, Pichiorri F, et al. Phase I trial of lenalidomide and CCI-779 in patients with relapsed multiple myeloma: evidence for lenalidomide-CCI-779 interaction via P-glycoprotein. J Clin Oncol. 2011;29(25):3427-3434.

61. Mahindra A, Richardson PG, Hari P, et al. Updated results of a phase I study of RAD001 in combination with lenalidomide in patients with relapsed or refractory multiple myeloma with pharmacodynamic and pharmacokinetic analysis [abstract]. Blood (ASH Annual Meeting Abstracts). 2010;116(21):3051.

62. Richardson P, Weber D, Mitsiades CS, et al. A phase I study of vorinostat, lenalidomide, and dexamethasone in patients with relapsed or relapsed and refractory multiple myeloma: excellent tolerability and promising activity in a heavily pretreated population [abstract]. Blood (ASHAnnual Meeting Abstracts). 2010;116(21):1951.

63. Richter JR, Bilotti E, McBride L, et al. Salvage therapy with vorinostat, lenalidomide, and dexamethasone (ZRD) in lenalidomide/dexamethasone relapsed/refractory multiple myeloma [abstract]. Blood (ASH Annual Meeting Abstracts). 2011;118(21):3986.

64. Jakubowiak AJ, Richardson PG, Zimmerman TM, et al. Final phase I results of perifosine in combination with lenalidomide and dexamethasone in patients with relapsed or refractory multiple myeloma (MM) [abstract]. Blood (ASH Annual Meeting Abstracts). 2010;116(21):3064.

65. Quach H, Ritchie D, Stewart AK, et al. Mechanism of action of immunomodulatory drugs (IMiDS) in multiple myeloma. Leukemia. 2010;24(1):22-32.

66. Hayashi T, Hideshima T, Akiyama M, et al. Molecular mechanisms whereby immunomodulatory drugs activate natural killer cells: clinical application. Br J Haematol. 2005;128(2):192-203.

67. van de Donk NW, Kamps S, Mutis T, Lokhorst HM. Monoclonal antibody-based therapy as a new treatment strategy in multiple myeloma. Leukemia. 2012;26(2):199-213.

68. Lonial S, Jakubowiak AJ, Jagannath S, et al. A phase 2 study of elotuzumab in combination with lenalidomide and low-dose dexamethasone in patients with relapsed/refractory multiple myeloma [abstract]. Blood (ASH Annual Meeting Abstracts). 2011;118(21):303.

69. Lonial S, Vij R, Harousseau JL, et al. Elotuzumab in combination with lenalidomide and low-dose dexamethasone in relapsed or refractory multiple myeloma. J Clin Oncol. 2012;30(16):1953-1959.

70. Berdeja JG, Ailawadhi S, Niesvizky R, et al. Phase I study of lorvotuzumab mertansine (IMGN901) in combination with lenalidomide and dexamethasone in patients with CD56-positive relapsed or relapsed/ refractory multiple myeloma - a preliminary safety and efficacy analysis of the combination [abstract]. Blood (ASH Annual Meeting Abstracts). 2010;116(21):1934.

71. Agura E, Niesvizky R, Matous J, et al. Dacetuzumab (SGN-40), lenalidomide, and weekly dexamethasone in relapsed or refractory multiple myeloma: multiple responses observed in a phase $1 \mathrm{~b}$ study [abstract]. Blood (ASH Annual Meeting Abstracts). 2009;114(22): 2870.

72. Zhang S, Liang J, Chen L, et al. Enhanced NK cell mediated cytotoxicity against multiple myeloma (MM) cells by the combination of anti-KIR (1-7F9) monoclonal antibody (mAb) and lenalidomide [abstract]. AACR Meeting Abstracts. 2009;(2_Annual_Meeting):3245.

73. Sakamaki I, Kaplan B, Cha S-C, Qin H, Kwak LW. Potent immunomodulatory effects of lenalidomide on effector T cells and Treg improve the effectiveness of a therapeutic lymphoma vaccine [abstract]. Blood (ASH Annual Meeting Abstracts). 2011;118(21):108.

74. Luptakova K, Glotzbecker B, Mills H, et al. Lenalidomide decreases PD-1 expression, depletes regulatory T-cells and improves cellular response to a multiple myeloma/dendritic cell fusion vaccine in vitro [abstract]. Blood (ASH Annual Meeting Abstracts). 2010;116(21): 492.

75. Noonan K, Rudraraju L, Ferguson A, et al. Lenalidomide-induced immunomodulation in multiple myeloma: impact on vaccines and antitumor responses. Clin Cancer Res. 2012;18(5):1426-1434. 
76. van de Donk NW, Kroger N, Hegenbart U, et al. Remarkable activity of novel agents bortezomib and thalidomide in patients not responding to donor lymphocyte infusions following nonmyeloablative allogeneic stem cell transplantation in multiple myeloma. Blood. 2006;107(8):3415-3416.

77. Kroger N, Badbaran A, Lioznov M, et al. Post-transplant immunotherapy with donor-lymphocyte infusion and novel agents to upgrade partial into complete and molecular remission in allografted patients with multiple myeloma. Exp Hematol. 2009;37(7):791-798.

78. Minnema MC, van der Veer MS, Aarts T, Emmelot M, Mutis T, Lokhorst HM. Lenalidomide alone or in combination with dexamethasone is highly effective in patients with relapsed multiple myeloma following allogeneic stem cell transplantation and increases the frequency of CD4+Foxp3+ T cells. Leukemia. 2009;23(3):605-607.

79. Kneppers E, van der Holt B, Kersten MJ, et al. Lenalidomide maintenance after nonmyeloablative allogeneic stem cell transplantation in multiple myeloma is not feasible: results of the HOVON 76 Trial. Blood. 2011;118(9):2413-2419.

80. El-Cheikh J, Crocchiolo R, Furst S, et al. Lenalidomide plus donorlymphocytes infusion after allogeneic stem-cell transplantation with reduced-intensity conditioning in patients with high-risk multiple myeloma. Exp Hematol. 2012;40(7):521-527.

81. Kumar SK, Rajkumar SV, Dispenzieri A, et al. Improved survival in multiple myeloma and the impact of novel therapies. Blood. 2008;111(5):2516-2520.

82. Palumbo A, Anderson K. Multiple myeloma. N Engl J Med. 2011; 364(11):1046-1060.

83. Palumbo A, Bringhen S, Ludwig H, et al. Personalized therapy in multiple myeloma according to patient age and vulnerability: a report of the European Myeloma Network (EMN). Blood. 2011;118(17): 4519-4529.

84. Quach H, Fernyhough L, Henderson R, et al. Lower-dose lenalidomide and dexamethasone reduces toxicity without compromising efficacy in patients with relapsed/refractory myeloma, who are aged $\geq 60$ years or have renal impairment: planned interim results of a prospective multicentre phase II trial [abstract]. Blood (ASH Annual Meeting Abstracts). 2010;116(21):1961.

85. Dimopoulos MA, Terpos E, GoldschmidtH,AlegreA, MarkT, Niesvizky R. Treatment with lenalidomide and dexamethasone in patients with multiple myeloma and renal impairment. Cancer Treat Rev. May 18, 2012. [Epub ahead of print.]

86. Niesvizky R, Naib T, Christos PJ, et al. Lenalidomide-induced myelosuppression is associated with renal dysfunction: adverse events evaluation of treatment-naive patients undergoing front-line lenalidomide and dexamethasone therapy. Br J Haematol. 2007;138(5): 640-643.

87. Dimopoulos M, Alegre A, Stadtmauer EA, et al. The efficacy and safety of lenalidomide plus dexamethasone in relapsed and/or refractory multiple myeloma patients with impaired renal function. Cancer. 2010;116(16):3807-3814

88. Kleber M, Ihorst G, Udi J, Koch B, Wasch R, Engelhardt M. Prognostic risk factor evaluation in patients with relapsed or refractory multiple myeloma receiving lenalidomide treatment: analysis of renal function by eGFR and of additional comorbidities by comorbidity appraisal. Clin Lymphoma Myeloma Leuk. 2012;12(1):38-48.

89. Dimopoulos MA, Christoulas D, Roussou M, et al. Lenalidomide and dexamethasone for the treatment of refractory/relapsed multiple myeloma: dosing of lenalidomide according to renal function and effect on renal impairment. Eur J Haematol. 2010;85(1):1-5.

90. de la Rubia J, Roig M, Ibanez A, et al. Activity and safety of lenalidomide and dexamethasone in patients with multiple myeloma requiring dialysis: a Spanish multicenter retrospective study. Eur J Haematol. 2010;85(4):363-365.

91. Palumbo A, Blade J, Boccadoro M, et al. How to manage neutropenia in multiple myeloma. Clin Lymphoma Myeloma Leuk. 2012;12(1): $5-11$.
92. Bennett CL, Angelotta C, Yarnold PR, et al. Thalidomide- and lenalidomide-associated thromboembolism among patients with cancer. JAMA. 2006;296(21):2558-2560.

93. Zonder JA, Barlogie B, Durie BG, McCoy J, Crowley J, Hussein MA. Thrombotic complications in patients with newly diagnosed multiple myeloma treated with lenalidomide and dexamethasone: benefit of aspirin prophylaxis. Blood. 2006;108(1):403; author reply 404.

94. Knight R, DeLap RJ, Zeldis JB. Lenalidomide and venous thrombosis in multiple myeloma. N Engl J Med. 2006;354(19):2079-2080.

95. Palumbo A, Rajkumar SV, Dimopoulos MA, et al; for International Myeloma Working Group. Prevention of thalidomide- and lenalidomide-associated thrombosis in myeloma. Leukemia. 2008; 22(2):414-423.

96. Larocca A, Cavallo F, Bringhen S, et al. Aspirin or enoxaparin thromboprophylaxis for patients with newly diagnosed multiple myeloma treated with lenalidomide. Blood. 2012;119(4):933-939; quiz 1093.

97. Lonial S, Richardson PG, San Miguel J, et al. Characterisation of haematological profiles and low risk of thromboembolic events with bortezomib in patients with relapsed multiple myeloma. Br J Haematol. 2008;143(2):222-229.

98. Pineda-Roman M, Zangari M, van Rhee F, et al. VTD combination therapy with bortezomib-thalidomide-dexamethasone is highly effective in advanced and refractory multiple myeloma. Leukemia. 2008;22(7):1419-1427.

99. Ciolli S, Leoni F, Casini C, Breschi C, Santini V, Bosi A. The addition of liposomal doxorubicin to bortezomib, thalidomide and dexamethasone significantly improves clinical outcome of advanced multiple myeloma. Br J Haematol. 2008;141(6):814-819.

100. Ciolli S, Leoni F, Gigli F, Rigacci L, Bosi A. Low dose Velcade, thalidomide and dexamethasone (LD-VTD): an effective regimen for relapsed and refractory multiple myeloma patients. Leuk Lymphoma. 2006;47(1):171-173.

101. Palumbo A, Ambrosini MT, Benevolo G, et al; for Italian Multiple Myeloma Network, Gruppo Italiano Malattie Ematologicche dell'Adulto. Bortezomib, melphalan, prednisone, and thalidomide for relapsed multiple myeloma. Blood. 2007;109(7):2767-2772.

102. Terpos E, Kastritis E, Roussou M, et al. The combination of bortezomib, melphalan, dexamethasone and intermittent thalidomide is an effective regimen for relapsed/refractory myeloma and is associated with improvement of abnormal bone metabolism and angiogenesis. Leukemia. 2008;22(12):2247-2256.

103. Zangari M, Fink L, Zhan F, Tricot G. Low venous thromboembolic risk with bortezomib in multiple myeloma and potential protective effect with thalidomide/lenalidomide-based therapy: review of data from phase 3 trials and studies of novel combination regimens. Clin Lymphoma Myeloma Leuk. 2011;11(2):228-236.

104. Zangari M, Tricot G, Polavaram L, et al. Survival effect of venous thromboembolism in patients with multiple myeloma treated with lenalidomide and high-dose dexamethasone. J Clin Oncol. 2010;28(1):132-135.

105. Rajkumar SV, Hayman SR, Lacy MQ, et al. Combination therapy with lenalidomide plus dexamethasone (Rev/Dex) for newly diagnosed myeloma. Blood. 2005;106(13):4050-4053.

106. Klein U, Kosely F, Hillengass J, et al. Effective prophylaxis of thromboembolic complications with low molecular weight heparin in relapsed multiple myeloma patients treated with lenalidomide and dexamethasone. Ann Hematol. 2009;88(1):67-71.

107. Palumbo A, Delforge M, Catalano J, et al. A phase 3 study evaluating the efficacy and safety of lenalidomide combined with melphalan and prednisone In patients $\geq 65$ years with newly diagnosed multiple myeloma (NDMM): continuous use of lenalidomide vs fixed-duration regimens [abstract]. Blood (ASH Annual Meeting Abstracts). 2010;116(21):622.

108. Palumbo A, Hajek R, Delforge M, et al; for MM-015 Investigators Continuous lenalidomide treatment for newly diagnosed multiple myeloma. N Engl J Med. 2012;366(19):1759-1769. 
109. Attal M, Lauwers Vc, Marit G, et al. Maintenance treatment with lenalidomide after transplantation for MYELOMA: final analysis of the IFM 2005-2002 [abstract]. Blood (ASHAnnual Meeting Abstracts). 2010;116(21):310.

110. McCarthy P, Owzar K, Anderson K, et al. Phase III intergroup study of lenalidomide versus placebo maintenance therapy following single autologous stem cell transplant (ASCT) for multiple myeloma (MM): CALGB ECOG BMT-CTN 100104. Haematologica. 2011;96(s1):S23.

111. Attal M, Lauwers-Cances V, Marit G, et al; for IFM Investigators. Lenalidomide maintenance after stem-cell transplantation for multiple myeloma. N Engl J Med. 2012;366(19):1782-1791.

112. McCarthy PL, Owzar K, Hofmeister CC, et al. Lenalidomide after stem-cell transplantation for multiple myeloma. N Engl J Med. 2012; 366(19):1770-1781.

113. Dimopoulos MA, Richardson PG, Brandenburg N, et al. A review of second primary malignancy in patients with relapsed or refractory multiple myeloma treated with lenalidomide. Blood. 2012;119(12):2764-2767.
114. Dimopoulos MA, Palumbo A, Attal M, et al; for European Myeloma Network. Optimizing the use of lenalidomide in relapsed or refractory multiple myeloma: consensus statement. Leukemia. 2011;25(5):749-760.

115. Barosi G, Merlini G, Billio A, et al. SIE, SIES, GITMO evidencebased guidelines on novel agents (thalidomide, bortezomib, and lenalidomide) in the treatment of multiple myeloma. Ann Hematol. 2012;91(6):875-888.

116. Kumar SK, Lee JH, Lahuerta JJ, et al; for International Myeloma Working Group. Risk of progression and survival in multiple myeloma relapsing after therapy with IMiDs and bortezomib: a multicenter international myeloma working group study. Leukemia. 2012;26(1):149-157.

117. van de Donk NW, Lokhorst HM, Dimopoulos M, et al. Treatment of relapsed and refractory multiple myeloma in the era of novel agents. Cancer Treat Rev. 2011;37(4):266-283.
Cancer Management and Research

\section{Publish your work in this journal}

Cancer Management and Research is an international, peer-reviewed open access journal focusing on cancer research and the optimal use of preventative and integrated treatment interventions to achieve improved outcomes, enhanced survival and quality of life for the cancer patient. The journal welcomes original research, clinical \& epidemiological

\section{Dovepress}

studies, reviews \& evaluations, guidelines, expert opinion \& commentary, case reports \& extended reports. The manuscript management system is completely online and includes a very quick and fair peerreview system, which is all easy to use. Visit http://www.dovepress.com/ testimonials.php to read real quotes from published authors. 